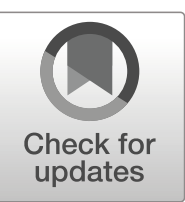

\title{
Correction to: Risk Factors and Complications for Revision Shoulder Arthroplasty
}

Natalie L. Leong, MD • Shelby Sumner, MPH • Anirudh K. Gowd, BS • Gregory P. Nicholson, MD • Anthony A. Romeo, MD • Nikhil N. Verma, MD

Published online: 1 May 2019

(C) Hospital for Special Surgery 2019

\section{Correction to: HSSJ 2019.}

https://doi.org/10.1007/s11420-019-09673-3

The name of author Anirudh K. Gowd was listed incorrectly in the original article. It is corrected here.

The online version of the original article can be found at https://doi.org/ 10.1007/s11420-019-09673-3

N. L. Leong, MD $(\bowtie)$

University of Maryland Medical Center,

300 S. Paca St.,

Baltimore, MD 21201, USA

e-mail: nleong@som.umaryland.edu

N. L. Leong, MD

Baltimore Veterans Administration Medical Center,

10 N. Greene St.,

Baltimore, MD 21201, USA

S. Sumner, MPH • A. K. Gowd, BS • G. P. Nicholson, MD •

N. N. Verma, MD

Rush University Medical Center,

1611 West Harrison Street, Suite 300,

Chicago, IL 60612, USA

\section{A. A. Romeo, MD}

Rothman Institute Orthopaedics,

176 3rd Ave,

New York, NY, USA 